\title{
Performance analysis of SAC based non-coherent optical CDMA system for OOC with variable data rates under noisy environment Indu Bala ${ }^{1}$ and Vanita Rana ${ }^{2}$ \\ ${ }^{1}$ Chandigarh Engg. College, Mohali (PB)-140307; ' Jaypee Univ. of Info. Technol., Waknaghat, Solan (H.P)-173215, India indu469@gmail.com; vanita.rana@juit.ac.in
}

Abstract: Simulation has become a tool in analyzing, optimizing and designing of optical networks. In this paper, simulated work has been presented for SAC based incoherent optical CDMA system with variable data rates for projective geometry based $\mathrm{OOC}$ in a C- band of available optical spectrum. System performance has been analyzed using Gaussian approximation method for different code parameters with variable data rates to support different LAN applications. In addition, major physical impairment factors such as shot noise, thermal noise, phase induced intensity noise, optical power received per user and multiple user interference have been considered in simulations.

Keywords: Optical orthogonal codes, spectral amplitude coding, bit error rate, phase induced intensity noise, multiple user interface.

Introduction

The biggest technical challenge for today's communication network systems is to take more information carrying capacity since the volume of information produced increases rapidly (Wei et al., $2002 a, b)$. Between the years 2000-2006 the volume of generated data grew from 3 billion to 160 billion Gigabytes. With the substantial growth in data traffic, the need for higher capacity optical systems increases.

Optical code division multiplexing access (OCDMA) is one class of such promising technology that takes advantage of excess bandwidth in single mode optical fiber to map low information rate into high rate optical sequences followed by optical transmitter to obtain random, asynchronous access without centralized control among users (Salehi, 1989; Salehi \& Brackett, 1989; Salehi et al., 1989).

The optical CDMA has attracted much attention for various applications of optical communication due to its advantageous features like simple, random and simultaneous access protocol, suitability for multimedia applications, security, no need of strict timing and wavelength control, effective utilization of bandwidth, immunity to various noises etc. (Prucnal et al.,1986).

A typical Optical CDMA communication network in star configuration is shown in Fig. 1. Users communicate by imprinting their message bits upon their unique address sequences, which they transmit asynchronously over a common channel. Discrimination between them is achieved by assigning minimally interfering spreading sequences to each user, selected from a family of so called optical orthogonal codes (OOC's).

Optical orthogonal codes for OCDMA system

The key to an effective CDMA system is the choice of efficient address code sequences with excellent autocorrelation and cross correlation properties for encoding and decoding of the source bits (Kwong et al., 1991). An optical orthogonal code (OOC) is a family of $(0$, 1) sequence with good auto and cross correlation properties. The auto correlation amplitude must be very high and the cross correlation and in phase cross correlation amplitude between two sequences must remain as low as possible (Salehi, 1989). The high value of auto correlation amplitude facilitates the detection of the desired signal and low cross correlation amplitude helps in reducing interference form users. Extensive research has been made to find out the suitable optical orthogonal codes for non-coherent optical CDMA systems.

In our previous work (Indu Bala \& Gurpal Singh, 2008), integer lattice design based SAC optical orthogonal code $(\mathrm{OOC})$ for fast frequency-hopping and time-spreading schemes was analyzed. Its performance was evaluated for number of simultaneous users, effective optical power and different code parameters ( $m$ \& k) using Gaussian approximation. In this paper, the performance of the same code has been analyzed for variable data rates and for different noise conditions.

\section{System performance analysis}

In this section, signal to noise ratio (SNR) and bit error rate (BER) performance for incoherent SAC optical CDMA has been investigated for integer lattice design based optical orthogonal code using method suggested by Wei et al. (2001). It has been assumed that multi user interference (MUI) is mainly due to thermal noise, shot noise and phase induced intensity noise (PIIN). The effect of dark current for photodiodes is negligible and therefore has been neglected here.

$$
S N R=\frac{\frac{R^{2} P s r^{2}}{P^{2}}}{q \Delta f R P s \cdot\left[\frac{k+(\lambda+1)(N-1)}{k}\right]+\frac{\Delta f R^{2} P s r^{2} k N}{2 p^{2} k^{2} \Delta f c}\left[\frac{N-1}{k-\lambda}+k+\lambda(N-1)\right]+\frac{4 k_{b} T r \Delta \mathcal{A}_{n} F_{n}}{R_{L}}}
$$

and

$$
\begin{aligned}
& B E R=\frac{1}{2} \operatorname{erfc}\left(\sqrt{\frac{S N R}{8}}\right) \quad \text { where } \\
& \operatorname{erfc}(x)=\frac{2}{\sqrt{\pi}} \int_{x}^{\infty} e^{-u^{2}} \cdot d u
\end{aligned}
$$

Fig. 2- 4 shows variations in BER with respect to number of simultaneous users at different effective power $P_{s r}$ from each user. The simulations have been done at variable data rates. It is clear from these figures that if we try to increase the data rate from each user to exploit the full potential of single mode optical fiber, it results severe degradation in system performance.
Research article

CIndian Society for Education and Environment (iSee)
"Optical code division multiplexing access" http://www.indjst.org
Indu \& Vanita Indian J.Sci.Technol. 
Fig 1. Optical CDMA communication network in star configuration with "N" transmitters/ receivers

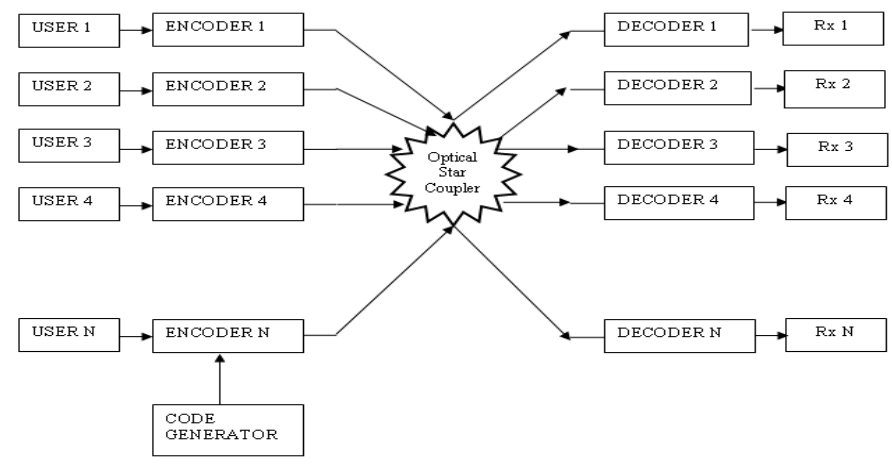

Fig. 3. BER performance for 25 simultaneous users with $m=37$ and $k=19$ for variable data rates at $-5 \mathrm{dBm}$

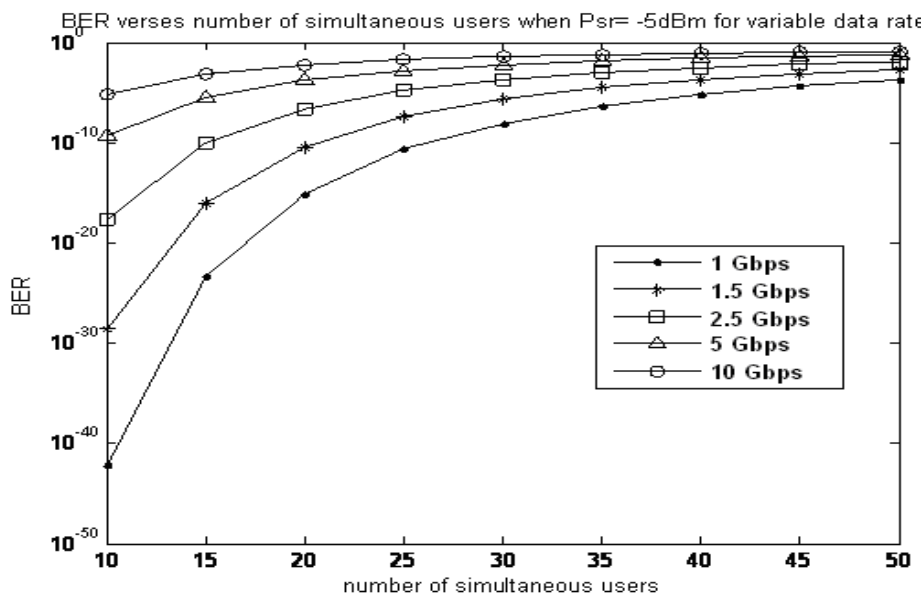

Fig.5. Effect of Shot noise on Bit Error Rate performance at $P_{s r}=-20 \mathrm{dBm} \&-30 \mathrm{dBm}$ with $160 \mathrm{Mbps}$ data rate for $m=37$ and $k=19$

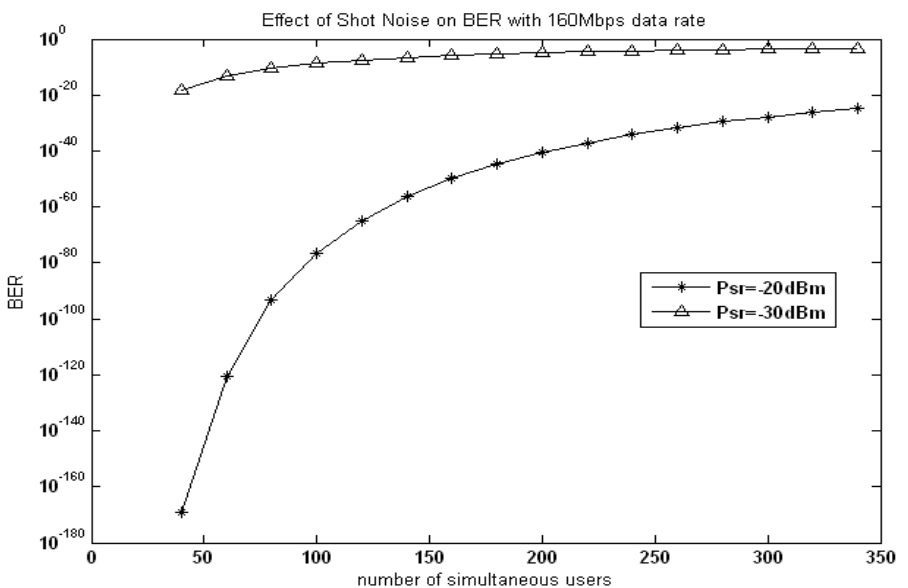

Vol.2 No. 8 (Aug 2009)

ISSN: 0974- 6846

Fig. 2. BER performance for 50 simultaneous users with $m=37$ and $k=19$ for variable data rates at $-10 \mathrm{dBm}$.

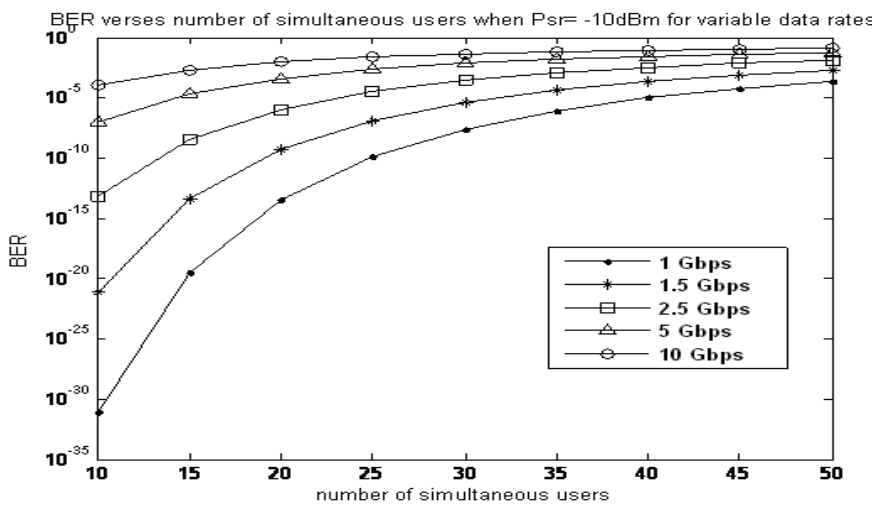

Fig. 4. BER performance for 25 simultaneous users with $m=37$ and $k=19$ for variable data rates at $-20 \mathrm{dBm}$.

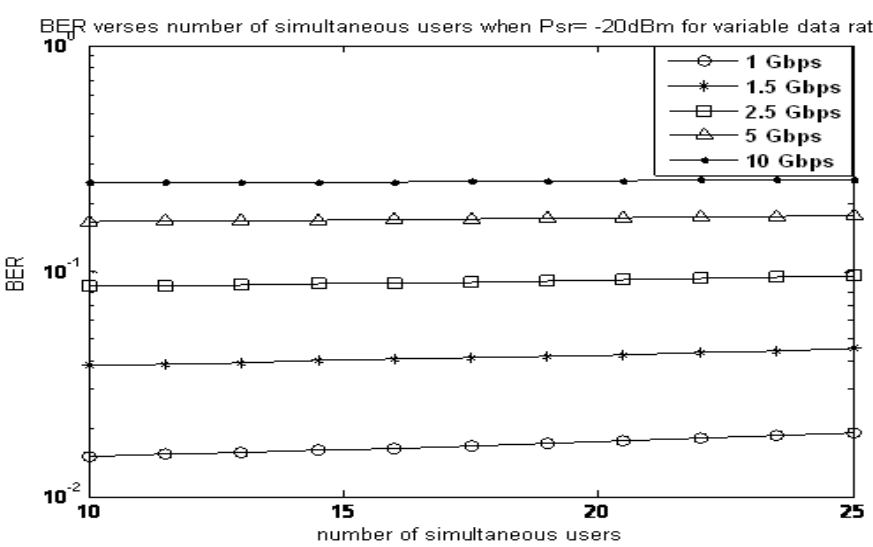

Fig. 6. Effect of shot noise on BER with $m=37$ and $k=19$ at $P_{s r}=-20 \mathrm{dBm}$ for variable data rates

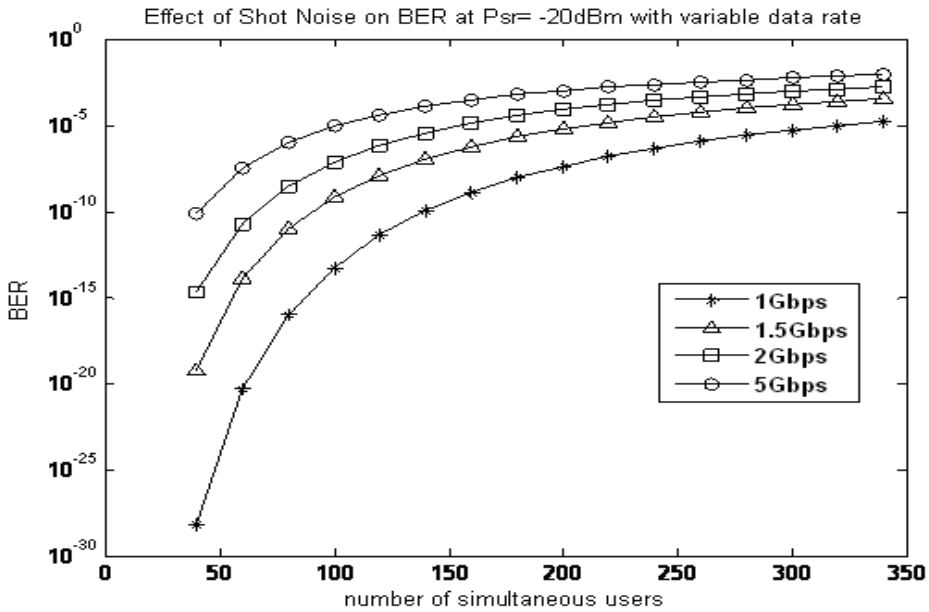

Research article

(C)Indian Society for Education and Environment (iSee)
"Optical code division multiplexing access" http://www.indjst.org
Indu \& Vanita Indian J.Sci.Technol. 
Indian Journal of Science and Technology

Fig. 7. Effect of PIIN noise on BER with $m=37$ and $k=19$ at $P_{s r}=-20 \mathrm{dBm}$ for $160 \mathrm{Mbps}$ data rate

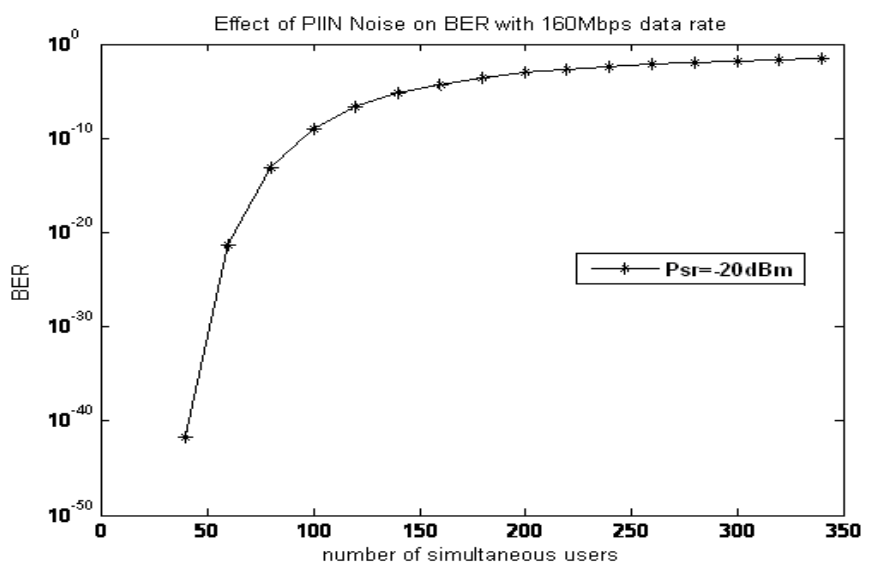

Fig. 9. Effect of Shot noise on BER with $m=37$ and $k=19$ at $P_{s r}=-10 \mathrm{dBm}$ for variable data rates.

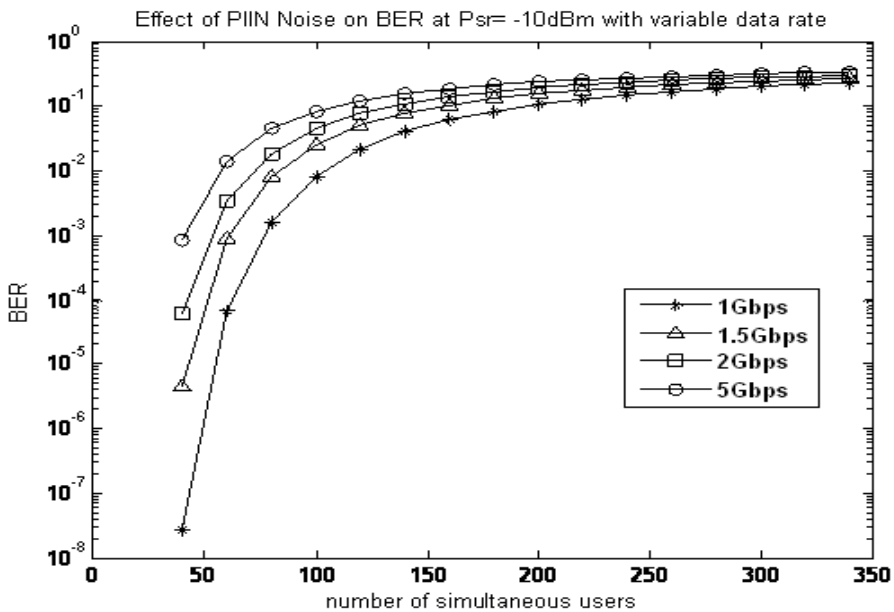

Fig. 11. BER variations with respect to effective user power when subjected of different noises for 121 simultaneous users with $m=37$ and $k=19$ at 160 Mbps.

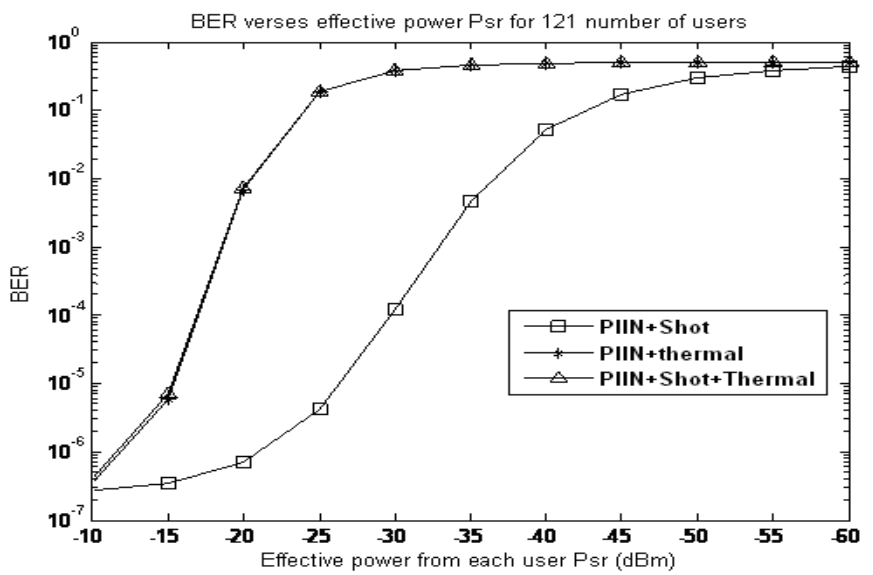

Fig.8. Effect of PIIN noise on BER with $m=37$ and $k=19$ at $P_{s r}=-20 \mathrm{dBm}$ for variable data rates

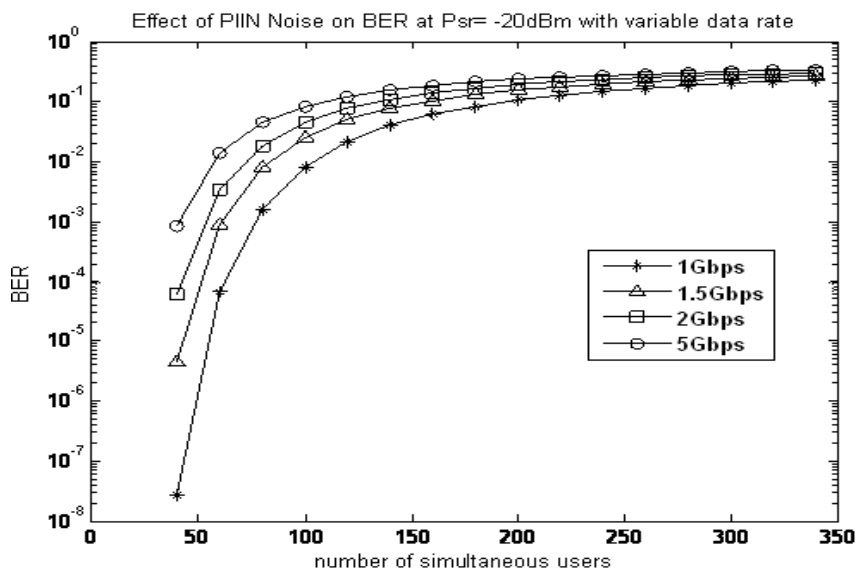

Fig. 10. BER variations with respect to effective user power when subjected of different noises for 49 simultaneous users with $m=37$ and $k=19 a t 160 \mathrm{Mbps}$.

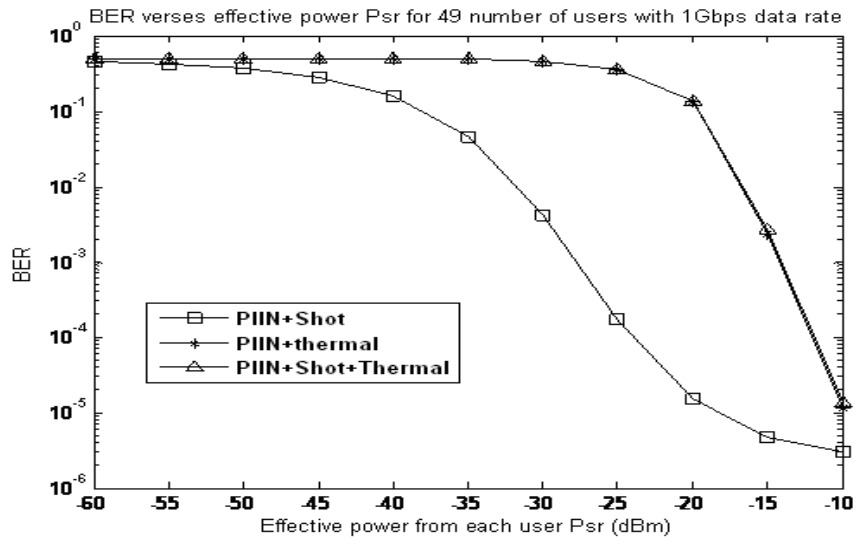

Fig. 12. BER variations with respect to effective user power when subjected of different noises for 49 simultaneous users with $m=37$ and $k=19$ at 1 Gbps

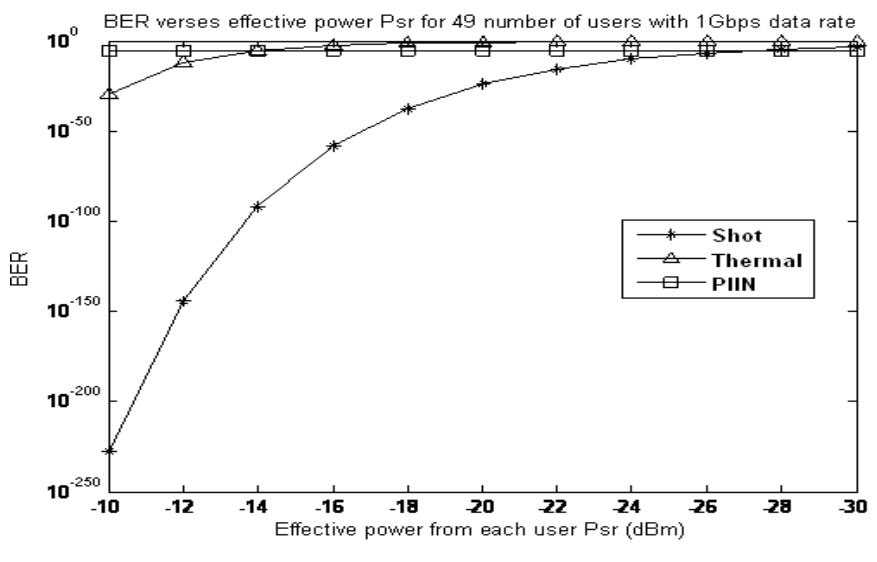

Research article CIndian Society for Education and Environment (iSee)
"Optical code division multiplexing access" http://www.indjst.org
Indu \& Vanita Indian J.Sci.Technol. 
The effect of an effective optical power from each user can also be observed from these figures. When $P_{s r}$ is more than $-10 \mathrm{dBm}$, the system performance improves significantly. This is because, with large $P_{\text {sr }}$, the effect of both shot noise and thermal noise is negligible as compare to the phase induced intensity noise (PIIN).

Fig. 5 is showing the effect of shot noise on BER at different $P_{s r}$ to strengthen the statement made above. Shot noise effect on system performance is more pronounced when $P_{s r}$ is small. Fig. 6 is showing effect of variable data rate on system performance under the influence of shot noise alone. It is clear from the figure that with increase in data rate, BER also increases. With low data rates more number of simultaneous users can be supported by a system, which definitely affects the quality of service offered by the system. With higher data rates comparatively less number of users can be supported. Fig. 7- 9 are showing the effect of PIIN alone on BER of the system with variable data rates. The effect of an effective optical power from each user can also be observed here. If we compare the Fig. 7 with Fig. 5, it becomes apparent that PIIN is main limitation factor to the system performance in comparison with the shot noise. The noise is strongly related to the multiple access interferences due to the overlapping of the optical spectra from different users. The effect is easily noticeable from the steepness of the graphs with simultaneous increase in the number of simultaneous users and data rates. By comparing Fig. 8 and 9, we can easily make out that increase in the effective power from each user does not help much to overcome the PIIN noise.

The effect of PIIN can only be reduced by designing new code sequences for the SAC based non coherent optical systems with minimum or zero cross correlation properties. Fig. 10 and 11 show the BER variations with respect to the effective optical power $P_{s r}$ from each user. The simulations have been done for different noise combinations when system is operated at $160 \mathrm{Mbps}$ with code parameters $\mathrm{m}=37$ and $\mathrm{k}=19$ for 49 and 121 simultaneous users respectively. Fig. 12 is showing the $B E R$ variations with respect to the $P_{s r}$ for 49 simultaneous users when operated at $1 \mathrm{Gbps}$. Form these graphs it is clear that the effect of noises become more pronounced as the number of simultaneous users increases. When $\mathrm{P}_{\mathrm{sr}}$ is large, both, shot noise and thermal noises are negligible as compare to the phase induced intensity noise. Whereas, with small $P_{s r}$, the effect of phase induced intensity noise become minimum and thermal noise causes degradation in system performance.

Conclusion

In this paper, the system performance for SAC based non coherent optical CDMA system has been evaluated by using Gaussian approximation at variable data rate. It has been observed that on increasing data rate from each user, cause severe degradation in system performance. With low data rates more number of simultaneous users can be supported by a system, but there is a tradeoff between numbers of simultaneous users and the QOS offered by the system. With higher data rates comparatively less number of users can be supported.

When $P_{\text {sr }}$ is large $(>-10 \mathrm{dBm})$, PIIN is main limitation factor to the system performance as compare to the shot noise and thermal noise. However, when $P_{s r}$ is low, thermal noise source becomes the main factor that limits the system performance. It is also been observed that thermal noise is much more influential than shot noise.

\section{References}

1. Azizoglu M, Salehi JA and Li Y (1992) Optical CDMA via temporal codes. IEEE Trans. Commun. 40, 11621170.

2. Indu Bala and Gurpal Singh (2008) Performance considerations for integer lattice design based OOC for incoherent optical CDMA system. ICTON 2008. pp: 58-61.

3. Kwong WC, Perrier PA and Prucnal PR (1991) Performance comparison of asynchronous and synchronous code-division multiple-access techniques for fibre-optical local area networks. IEEE Trans. Commun. 39, 1625-1634.

4. Prucnal PR, Santoro MA and Sehgal S K (1986) Ultrafast all-optical synchronous multiple access fibre networks. IEEE J. Select. Areas Commun. SAC-4, 1484-1493.

5. Salehi JA (1989) Code division multiple access techniques in optical fiber networks-part I: Fundamental principles. IEEE Commun. 37, 824-833.

6. Salehi JA and Brackett CA (1989) Code division multiple access techniques in optical fiber networkspart II: system performance analysis. IEEE Trans. on Commun. 37, 834-842.

7. Salehi JA, Chung FRK and Wei VK (1989) Optical orthogonal codes: design, analysis, and applications. IEEE Trans. on Info. Theory. 35, 595-605.

8. Wei Z, Shalaby HMH and Ghafouri-Shirz H (2001) Modified quadratic congruence codes for fibre Bragg grating based spectral amplitude coding optical CDMA systems. IEEE J. Lightwave Technol. 19, 1274-1281.
Research article

CIndian Society for Education and Environment (iSee)
"Optical code division multiplexing access" http://www.indjst.org
Indu \& Vanita Indian J.Sci.Technol. 